Revue internationale P.M.E.

Économie et gestion de la petite et moyenne entreprise

\title{
Information financière et politique d'offre de crédit bancaire aux PME : cas du Cameroun
}

\section{Henri Wamba et Louise Tchamanbé-Djiné}

Volume 15, numéro 1, 2002

URI : https://id.erudit.org/iderudit/1008802ar

DOI : https://doi.org/10.7202/1008802ar

Aller au sommaire du numéro

Éditeur(s)

Presses de l’Université du Québec

ISSN

0776-5436 (imprimé)

1918-9699 (numérique)

Découvrir la revue

Citer cet article

Wamba, H. \& Tchamanbé-Djiné, L. (2002). Information financière et politique d'offre de crédit bancaire aux PME : cas du Cameroun. Revue internationale P.M.E., 15(1), 87-114. https://doi.org/10.7202/1008802ar

\section{Résumé de l'article}

L'information financière est un élément déterminant pour la banque dans l'appréciation du risque de l'emprunteur. Elle soulève un certain nombre de problèmes, notamment celui de son imperfection due à l'asymétrie d'information. La littérature financière propose un ensemble de signaux et mécanismes incitatifs susceptibles de réduire l'imperfection de l'information qui existe entre le créancier et l'emprunteur. Pour identifier ceux sur lesquels se fondent les banques camerounaises dans leur décision de prêts aux PME, une étude empirique a été réalisée. Il en est ressorti que, lorsque la relation de clientèle ou relation de long terme appréhendée par la régularité dans le remboursement des prêts n'est pas prise en compte, l'apport personnel, la valeur de la garantie et le ratio d'autonomie financière traduisant de manière générale l'importance du patrimoine de l'emprunteur sont les signaux financiers déterminants devant le niveau d'engagement envers les tiers et la rentabilité du projet. Lorsque la relation de clientèle est prise en considération dans le test, le patrimoine de l'emprunteur est relégué au second plan au profit de cette dernière. Il est apparu par ailleurs que le dividende versé aux actionnaires a une portée significative limitée dans la décision du banquier, compte tenu de la structure du capital « unipersonnel » de la plupart des PME camerounaises.
Ce document est protégé par la loi sur le droit d'auteur. L'utilisation des services d'Érudit (y compris la reproduction) est assujettie à sa politique d'utilisation que vous pouvez consulter en ligne.

https://apropos.erudit.org/fr/usagers/politique-dutilisation/ 


\title{
Information financière et politique d'offre de crédit bancaire aux PME: cas du Cameroun 1
}

\author{
Henri WAMBA \\ Louise TCHAMANBÉ-DJINÉ \\ Université de Yaoundé II
}

MOTS CLÉS

\section{Aléa moral - Asymétrie d'information - Crédit bancaire Information financière - Mécanismes incitatifs Risque de défaut - Sélection adverse}

\begin{abstract}
RÉSUMÉ
L'information financière est un élément déterminant pour la banque dans l'appréciation du risque de l'emprunteur. Elle soulève un certain nombre de problèmes, notamment celui de son imperfection due à l'asymétrie d'information. La littérature
\end{abstract}

1. Nous tenons à remercier les examinateurs et particulièrement l'examinateur $n^{0} 2$ pour ses observations pertinentes, lesquelles nous ont permis d'améliorer profondément les deux premières versions de notre article.

\section{LES AUTEURS}

HENRI WAMBA détient un doctorat en sciences de gestion de l'Université de Rennes I (France). En plus d'être enseignant-chercheur et directeur du GRFIC (Groupe de recherche en finance et comptabilité) à la Faculté des sciences économiques et de gestion de l'Université de Yaoundé II (Cameroun), il est chercheur associé au LARGOR (Laboratoire de recherche en gestion des organisations de Rennes), IAE-IGR, URA-CNRS 1418. Adresse : Université de Yaoundé II, Faculté des sciences économiques et de gestion, B.P. 13544, Yaoundé, Cameroun. Courriel <wamba.orbit@cenadi.cm>.

LOUISE TCHAMANBÉ-DJINÉ possède un doctorat en économie monétaire, financière et bancaire de l'Université Montesquieu, Bordeaux IV (France). Elle est enseignante-chercheure à la Faculté des sciences économiques et de gestion de l'Université de Yaoundé II (Cameroun) et membre de l'Équipe n 4 du réseau Entrepreneuriat de l'AUF (Orléans). Adresse : Université de Yaoundé II, Faculté des sciences économiques et de gestion, B.P. 13544, Yaoundé, Cameroun. Courriel <ltchamanbe@yahoo.fr>. 
financière propose un ensemble de signaux et mécanismes incitatifs susceptibles de réduire l'imperfection de l'information qui existe entre le créancier et l'emprunteur. Pour identifier ceux sur lesquels se fondent les banques camerounaises dans leur décision de prêts aux PME, une étude empirique a été réalisée. II en est ressorti que, lorsque la relation de clientèle ou relation de long terme appréhendée par la régularité dans le remboursement des prêts n'est pas prise en compte, l'apport personnel, la valeur de la garantie et le ratio d'autonomie financière traduisant de manière générale l'importance du patrimoine de l'emprunteur sont les signaux financiers déterminants devant le niveau d'engagement envers les tiers et la rentabilité du projet. Lorsque la relation de clientèle est prise en considération dans le test, le patrimoine de l'emprunteur est relégué au second plan au profit de cette dernière. Il est apparu par ailleurs que le dividende versé aux actionnaires a une portée significative limitée dans la décision du banquier, compte tenu de la structure du capital « unipersonnel » de la plupart des PME camerounaises.

\begin{abstract}
The financial information is an element determining for the bank in the assessment of the risk of the borrower. It lifts a certain number of problems, notably that of its imperfection due to the asymmetry of information. The financial literature proposes a set of signals and mechanisms and incentives susceptible to reduce the imperfection of the information that exists between the lender and the borrower. To identify those on which Cameroonian banks base their decision of loans for the small businesses, an empirical study has been realized. It emerged that, when the customer relationship or the long term relation apprehended by the regularity in the refund of loans is not taken into account, the personal capital, the value of the collateral and the ratio of financial autonomy translating in general terms the importance of the capital of the borrower are financial signals determining ahead the level of commitment towards third parties and the profitability of the project. When the relationship of clientele is taken into consideration in the test, the capital of the borrower is relegated to the second plan to the profit of this last. It appeared furthermore that the versed dividend to shareholders has a limited significant reach in the decision of the banker considering the structure of the capital "plainpersonnel» of most Cameroonian small businesses.
\end{abstract}

\title{
RESUMEN
}

La información financiera es un elemento de gran relevancia para el banco en cuanto a la apreciación del riesgo del prestatario. Plantea muchos problemas como él de su imperfección debida a la asimetría de información. La literatura financiera propone un conjunto de señales y mecanismos incitativos aptos para disminuir la imperfección de la información que existe entre el acreedor y el prestatario. Para identificar las personas en quienes se fundan los bancos cameruneses en lo concerniente a su decisión de los préstamos a los pequeñas empresas, un estudio empírico ha sido realizado. Se ha concluido que cuando la relación de clientela o la relación de plazo temida por la frecuencia en el reembolso de los préstamos no es tomada en cuenta, la oportación personal, el valor de la garantía y el ratio de autonomía financiera, traduciendo de manera general la importancia del patrimonio del prestatario, son los señales financieros determinantes en cuanto al nivel del

Revue internationale P.M.E., vol. 15, $\mathrm{n}^{\circ} 1,2002$ 
compromiso para con los tercios y la rentabilidad del proyecto. Cuando la relación de clientela es tomada en consideración en el test, el patrimonio del prestatario es confinado en el segundo término en provecho de ésta. Por otra parte, resultó que el dividendo desembolsado a los accionistas tenía un alcance significativo limitado por la decisión del banquero, teniendo en cuenta la estructura del capital " uni-personal » de la mayor parte de los pequeñas empresas.

\section{ZUSAMMENFASSUNG}

Die finanzielle Information ist ein entscheidendes Element für die Banken in der Risikoeinschätzung vom Kapitalnehmer. Sie ruft eine gewisse Anzahl von Problemen hervor, in erster Linie das Problem der Ungenauigkeit der Information, hervorgerufen durch ihre Asymetrie. Die französische Literatur schlägt deshalb Signale und Mechanismen vor, welche die ungenauen Informationen zwischen dem Kapitalgeber und dem Kapitalnehmer verringern. Um diejenigen Signale und Mechanismen zu identifizieren, auf welche sich die Banken aus Kamerun bei ihrer Darlehensentscheidung stützen, wurde eine empirische Untersuchung durchgeführt.

Dabei zeigte sich, dass wenn die Kundenbeziehung oder eine längerfristige, regelmässige Zurückzahlung der Schulden nicht berücksichtigt wird, sind die persönliche Einlage, der Garantiewert, das Verhältnis der finanziellen Unabhängigkeit und das Vermögen des Kapitalnehmers die entscheidenden finanziellen Signale sind, noch vor der Projektrentabilität.

\section{Introduction}

Depuis le début des années 1980, l'économie de la plupart des pays africains au sud du Sahara a connu une forte récession caractérisée par un très faible taux de croissance. Le ralentissement des activités s'est notamment manifesté dans le cas du Cameroun par un faible taux de création d'entreprises et de nombreux dépôts de bilans de plusieurs PME. Cette situation n'a pas épargné le système bancaire où l'on a enregistré un fort taux de faillites et de liquidations ${ }^{2}$. En effet, le marché bancaire camerounais est le plus important de la Communauté économique et monétaire de l'Afrique centrale (CEMAC) tant en volume de dépôts collectés qu'en montant de crédits octroyés. En 1997, année marquant la fin des restructurations bancaires au Cameroun, les concours bancaires représentent $43 \%$ de l'ensemble des six pays de la zone. La même tendance est observée jusqu'en 2000 avec une proportion de l'ordre de $47 \%$. De la même façon, les dépôts sont respectivement

2. Après les premières restructurations bancaires intervenues dès 1989,5 banques sur les 12 que comptait le système bancaire ont été liquidées. Malgré les nouvelles créations intervenues entre-temps, la deuxième phase de restructurations a mis fin aux activités de 6 banques sur les 11 existantes en 1997. Actuellement, le système bancaire camerounais compte 12 établissements de crédit parmi lesquels 4 sont des créations nouvelles.

Revue internationale P.M.E., vol. 15, n $\mathrm{n}^{\circ} 1,2002$ 
de $43 \%$ et de $48 \%$ au cours de la même période ; ce qui signifie que le Cameroun représente à lui seul plus de $40 \%$ de la part de marché de dépôts et de crédits des pays de la zone de la CEMAC. Lorsqu'on analyse la répartition des crédits par catégories socioprofessionnelles au cours de l'année 2000, il en ressort qu'environ $80 \%$ de ces concours sont distribués aux entreprises privées constituées pour l'essentiel de PME; ce qui représente un montant de plus de 394 millions de francs CFA. Malgré la présence sur le marché de structures de financement informelles (tontines) et la récente apparition des systèmes de financement décentralisés (notamment les coopératives d'épargne et de crédit) à la faveur de la loi de 1992 sur les associations, le système bancaire occupe encore une place prépondérante sur le marché du crédit. Cette position est sans doute justifiée par les effets positifs du vaste programme d'assainissement du réseau bancaire engagé dès 1989 : libéralisation des conditions de banque, création d'un marché monétaire, dévaluation du franc CFA... Toutefois, il convient de signaler que la proportion des crédits non remboursables par les PME reste encore considérable; celle-ci se situe autour de $20 \%$ de l'ensemble des crédits octroyés en l'an 2000, ce qui laisse penser que les effets de la crise bancaire sont encore d'actualité.

Les études menées sur les crises bancaires en Afrique (Le Noir, 1989, 1995 ; Rapport du groupe de travail ESF, 1990; Tamba et Tchamanbé-Djiné, 1995) ont révélé plusieurs causes, dont les plus importantes sont l'insuffisance des fonds propres des banques, la forte ingérence de l'État dans la gestion de celles-ci, les défaillances du système de gestion et le risque de défaut élevé des emprunteurs. Cette dernière cause, objet de notre préoccupation ici, aurait essentiellement pour origine les problèmes de gestion de l'information financière en matière de politique d'octroi de crédit bancaire.

L'information financière est un élément important pour la banque dans l'appréciation du risque de l'emprunteur et de sa capacité de remboursement. D'ordre quantitatif (comptable, mathématique et statistique) et qualitatif (marché, environnement) et technologique (sophistication des instruments et support de gestion), l'information financière soulève un certain nombre de problèmes liés, d'une part, à leur insuffisance et à leur imperfection et, d'autre part, à leur complexité. Ces aspects sont à l'origine des asymétries d'information (Sharpe, 1990) pouvant exister entre l'emprunteur et la banque et qui conduisent très souvent à la sélection adverse (Stiglitz et Weiss, 1981) et à l'aléa moral (Darrough et Stoughton, 1986; Chan et Thakor, 1987), phénomènes préjudiciables à l'appréciation du risque.

Dans une telle situation, comment surmonter l'insuffisance et l'imperfection de l'information financière pour adapter la politique d'offre de crédit aux besoins de financement des PME afin de limiter le risque de défaut? 
La littérature financière propose un ensemble de modèles : les modèles à signaux financiers (apport personnel de l'emprunteur-Leland et Pyle, 1977 ; structure financière de l'entreprise - Ross, 1977; versement des dividendes - Bhattacharya, 1979 ; Kalay, 1980 ; etc.) et les modèles à mécanismes incitatifs (garanties - Chan et Kanatos, 1985 ; Bester, 1987; Wette, 1983 ; relation de clientèle - Diamond, 1989, 1991 ; Sharpe, 1990 ; etc.), susceptibles de réduire les imperfections de l'information dans les relations de crédit.

Notre étude se propose, dans une première partie, de faire une analyse critique des modèles théoriques existants et, dans une deuxième partie, de tester empiriquement les conclusions de certains de ces modèles à partir de données camerounaises, ainsi que de certains signaux spécifiques au contexte de l'étude et susceptibles d'influencer la décision du banquier.

\section{Information financière et décision d'octroi de crédit bancaire : un aperçu théorique}

De manière générale, la décision d'octroi de crédit repose, si l'on se fie aux enseignements de base dans ce domaine, sur quatre critères, à savoir : les qualités morales et la compétence de l'emprunteur, l'importance des garanties pouvant être offertes, la capacité de remboursement de l'emprunteur et l'appréciation du risque financier. L'évaluation des deux derniers critères se fonde essentiellement sur la disponibilité de l'information financière. Cette information financière, qui se présente sous plusieurs formes, n'est pas sans poser de problèmes au banquier. Ces problèmes sont relatifs à leur insuffisance et à leur imperfection, ce qui oblige le banquier à disposer des moyens adéquats pour gérer cette situation. Après avoir énoncé les différents types d'information financière et leur importance (1.1.), nous examinerons les problèmes posés par celles-ci (1.2.) ainsi que les moyens utilisés pour réduire ses imperfections (1.3.).

\subsection{Différents types d'information financière et leur importance}

Il existe en théorie différents types d'information financière. Après avoir précisé le contenu de cette notion, on procédera à l'énumération des différentes formes sous lesquelles elle apparaît au sein d'une entreprise et qui peuvent servir au banquier pour prendre sa décision.

\subsubsection{Notion d'information financière}

L'information financière représente l'ensemble des données chiffrées ou non que l'on peut recueillir sur l'entreprise. Elle part de simples données comptables recueillies auprès des entreprises à travers les bilans et comptes de résultats, aux

Revue internationale P.M.E., vol. 15, $\mathrm{n}^{\circ}$ 1, 2002 
données fournies par l'environnement sur l'entreprise, notamment le marché, les partenaires commerciaux et sociaux, etc. Sur la base de cette considération et pour mieux appréhender cette notion, il y a lieu de l'examiner sous différents aspects.

\subsubsection{Types d'information financière et leurs supports}

L'information financière qui se présente sous forme quantitative et qualitative peut être aussi bien interne qu'externe à l'entreprise.

\section{a) L'information financière interne à l'entreprise}

L'information financière interne à l'entreprise regroupe essentiellement les données quantitatives issues des états financiers et comptables de l'entreprise : bilans, comptes de résultats et d'exploitation et, subsidiairement, les documents annexes donnant certains détails sur les échéances de dettes, les créances, le tableau d'amortissement des immobilisations de l'entreprise, etc. L'analyse de ces informations conduit à l'élaboration de données plus synthétiques telles que le fonds de roulement, les besoins en fonds de roulement, les ratios financiers, les tableaux de financement, etc., permettant d'apprécier la solvabilité de l'entreprise, sa rentabilité, sa capacité de remboursement ainsi que son risque financier.

C'est sur la base de ces données quantitatives que les banquiers ont élaboré un certain nombre de techniques permettant d'apprécier le risque de l'emprunteur, en l'occurrence la méthode de «credit scoring 》 (Altmann, 1980) ou méthode d'analyse discriminante.

\section{b) L'information financière externe à l'entreprise}

D'ordre quantitatif et qualitatif, l'information financière externe à l'entreprise regroupe les données en provenance des marchés financiers, des fournisseurs, du fisc, des organismes sociaux, de la centrale des risques, etc. Ces informations, très souvent subjectives et difficiles à recueillir, sont d'une importance fondamentale dans la décision du banquier. Une mauvaise appréciation de celles-ci par le banquier peut lui être préjudiciable, notamment en cas d'un éventuel redressement fiscal de l'entreprise ou d'un excès d'engagement de celle-ci auprès des fournisseurs. Ces différents aspects sont très souvent cause de défaillance de l'entreprise.

\section{c) Les supports de l'information financière}

Outre la comptabilité qui permet de concevoir des données financières plus élaborées pour le banquier, il existe d'autres supports de gestion de l'information financière. Il s'agit des moyens technologiques tels que l'informatique et la télématique 
(téléphone, télex, télécopieur, Internet, etc.). Vu la célérité de ces instruments, ils interviennent aussi bien au moment de la décision d'octroi de crédit que dans le suivi de l'emprunteur.

Quelle que soit la forme sous laquelle apparaît l'information financière, sa prise en compte dans la décision bancaire en matière d'octroi de crédit aux PME camerounaises soulève un certain nombre de problèmes.

\subsection{Problèmes posés par l'information financière}

Ces problèmes sont relatifs à l'insuffisance et à l'imperfection de l'information financière.

\subsubsection{Insuffisance de l'information financière}

Au niveau de l'information interne à l'entreprise, les banques camerounaises ont parfois du mal à collecter toutes les informations comptables et financières nécessaires, en raison du fait que très peu de PME tiennent régulièrement la comptabilité de leurs activités.

Sur le plan externe à l'entreprise, même si les banquiers ont les moyens d'obtenir des informations sur l'entreprise auprès du fisc, des organismes sociaux ainsi que de la centrale des risques, ils sont incapables de connaître exactement le niveau d'engagements de celle-ci à l'égard de ses fournisseurs et prêteurs informels (tontines et autres associations). Il y a lieu de préciser ici que la centrale des risques ne donne que le niveau d'engagements des entreprises à l'égard des institutions financières.

\subsubsection{Imperfection de l'information financière}

Outre les difficultés liées à l'insuffisance de l'information financière interne et externe à l'entreprise, d'autres sont liées à sa fiabilité.

Par ailleurs, en ce qui concerne les supports de l'information financière, il convient de signaler que dans le cadre camerounais, les entreprises n'ont toujours pas les moyens de se doter d'un outil informatique adéquat ni d'un personnel qualifié pour son utilisation. Aussi, le réseau télématique est peu fiable, ce qui réduit son champ d'utilisation.

La conséquence de cet état de choses est que le banquier n'arrive pas toujours à bien apprécier le risque de l'emprunteur. Pour remédier à cette situation, la théorie générale de la firme propose un certain nombre de solutions susceptibles de réduire les asymétries d'information entre le créancier et l'emprunteur.

Revue internationale P.M.E., vol. 15, nº 1, 2002 


\subsection{Moyens utilisés pour réduire les imperfections de l'information}

Les problèmes des imperfections de l'information font l'objet d'un débat qui date de plus de deux décennies et dont Akerlof (1970) est l'un des précurseurs avec son article sur l'étude des marchés d'occasion. Dans cet article, il montre que dans un contexte d'asymétrie d'information, le prix cesse d'être un indicateur de valeur; ce qui conduit très souvent aux problèmes de sélection adverse. Ce problème d'asymétrie d'information avec sélection adverse et aléa moral a été examiné dans le cadre des marchés de crédit où les principales contributions sont celles de Stiglitz et Weiss (1981, 1983), Chan et Kanatas (1985), Darrough et Stoughton (1986), Bester (1987), Diamond (1989, 1991) et Sharpe (1990). Ces travaux se répartissent dans deux grands groupes de modèles : les modèles à signaux financiers et les modèles à mécanismes incitatifs. À cela s'ajoutent les formules de capital-risque développées par différents auteurs notamment Wright et Robbie (1998). Ces deux auteurs montrent dans leurs travaux qu'en ce qui concerne le problème de financement, le capital-risque, domaine jusque-là négligé en finance d'entreprise et dont le champ d'application a été restreint aux seules entreprises innovatrices, peut être étendu à toute forme d'entreprise. L'avantage du capital-risque est lié au fait qu'en plus des informations financières relatives à l'entreprise, il tient compte des éléments non financiers, notamment les qualités de l'entrepreneur et ses compétences. Étant donné que les modèles à signaux financiers et ceux à mécanismes incitatifs courants dans la littérature financière sont le plus souvent développés pour les grandes entreprises, la prise en compte du capital-risque se révèle être une approche complémentaire dans le cas camerounais où l'information financière quantitative est souvent déficiente pour les PME; cela justifie le choix des variables testées dans la partie empirique de notre étude.

\subsubsection{Les modèles à signaux financiers}

Les modèles à signaux financiers ou modèles dits de « signalisation » sont ceux pour lesquels les promoteurs emprunteurs, mieux informés sur leurs projets, cherchent à signaler aux prêteurs les véritables caractéristiques de leurs entreprises. Un signal se définit comme une variable de comportement selon lequel les promoteurs de «bons » projets ont intérêt à rendre publiques les informations qui permettent aux créanciers de distinguer leurs projets des moins bons. Pour ce faire, ils vont utiliser une variable financière comme moyen de signalisation.

Parmi les signaux couramment évoqués dans la littérature, on peut mentionner l'importance de l'apport personnel en capital détenu par le promoteur dans son affaire (Leland et Pyle, 1977), la structure financière de l'entreprise (Ross, 1977) et l'importance des dividendes versés aux actionnaires (Bhattacharya, 1979, 1980; Kalay, 1980). 


\section{a) Signalisation par l'apport personnel en capital détenu par le promoteur}

Le modèle de référence en la matière est celui proposé par Leland et Pyle (1977); c'est un modèle d'équilibre simple de structure du capital et d'évaluation de la firme. L'idée soutenue par ces auteurs est que les promoteurs dirigeants actionnaires qui ont de meilleurs projets d'investissement le signalent aux éventuels créanciers par une proportion importante du capital investi dans leurs projets. Ce faisant, ils renoncent à la diversification de leur portefeuille qui représente un coût pour ces derniers et un bon signal pour les créanciers.

Par hypothèse, les promoteurs cherchent à financer les projets dont ils sont seuls à connaître la qualité, ce qui pose un problème d'asymétrie d'information. Leland et Pyle montrent que l'apport personnel du promoteur dans le projet peut servir comme signal de la qualité de projet étant donné que la valeur de la firme s'accroît avec la proportion de la participation détenue par le promoteur dans le capital de l'entreprise. Ainsi, il y aurait une relation positive et significative entre la valeur de l'entreprise et l'apport personnel en capital détenu par l'exploitant dans son projet.

\section{b) Signalisation par la structure financière de l'entreprise}

Les travaux les plus connus consacrés à l'aspect structure financière comme un moyen de limiter le risque de défaut de l'emprunteur sont ceux de Ross (1977).

Contredisant le théorème de Modigliani et Miller (1958) sur l'indifférence de la structure financière sur la valeur de l'entreprise, Ross montre que les managers définissent le type de la firme qu'ils dirigent par la structure financière qu'ils lui donnent. Ce qui découle des résultats empiriques du modèle de Ross est que la valeur de l'entreprise s'accroît avec le levier dans la mesure où l'augmentation de l'endettement entraîne un accroissement de la valeur perçue de la firme. Ainsi, partant d'un modèle simplifié avec deux catégories d'entreprises, puis en généralisant les résultats dans le cas où il y aurait une multitude d'entreprises de qualité différente, Ross arrive à la conclusion suivant laquelle l'augmentation de l'endettement ne fait pas qu'accroître le risque, mais signale aussi une plus grande productivité de l'entreprise. De ce fait, une corrélation apparaîtrait entre la valeur de la firme et le ratio d'endettement.

Cependant, il existe un seuil d'endettement optimal correspondant à la valeur la plus élevée de la firme au-delà duquel un endettement additionnel ferait baisser la valeur de l'entreprise, lui faisant courir ainsi un risque de défaillance élevé (Warner, 1977; Malécot, 1984). D'après Ross, le dépassement du seuil d'endettement optimal fait supporter au dirigeant une pénalité pouvant se traduire soit par la résiliation de son contrat, soit par la diminution ou la perte de certains avantages liés à sa fonction. Sur ce dernier point, Bhattacharya (1979) relève que, lorsque les

Revue internationale P.M.E., vol. 15, nº 1, 2002

(C) 2002 - Presses de l'Université du Québec

Édifice Le Delta I, 2875, boul. Laurier, bureau 450, Sainte-Foy, Québec G1V 2M2 • Tél. : (418) 657-4399 - www.puq.uquebec.ca

Tiré de : Revue internationale P.M.E., vol. $15, n^{\circ} 1$, sous la direction de Pierre-André Julien. 
coûts reposent uniquement sur le gestionnaire, l'incitation est forte et peut conduire à un faux signal. En effet, les actionnaires d'une entreprise à faible valeur peuvent amener le gestionnaire à émettre un faux signal permettant de surestimer la valeur de son entreprise afin de réaliser des gains en capital. Aussi préconise-t-il que les actionnaires assument, eux aussi, les coûts de faillite de l'entreprise due à un mauvais choix de sa structure financière.

\section{c) Signalisation par les dividendes versés aux actionnaires}

Considérer les dividendes comme un signal de la bonne qualité de l'emprunteur relève de la préoccupation d'un certain nombre d'auteurs. Les principaux travaux consacrés à la politique de dividendes comme signal du type de firme en présence sont ceux de Kalay (1980) et de Bhattacharya (1980).

Le modèle de Kalay se situe dans la même optique que celui de Ross. Ainsi, lorsque le dividende attendu est supérieur aux bénéfices de l'entreprise, le gestionnaire est dans l'impossibilité d'honorer ses engagements envers les actionnaires; il encourt ainsi une pénalité. Comme le modèle de Ross, il lui est reproché de faire supporter les coûts par les seuls gestionnaires alors que les actionnaires des moins bonnes entreprises sont en mesure d'inciter ces derniers à émettre de faux signaux de leur firme en contrepartie des «pourboires».

Partant de l'idée que l'imperfection de l'information est liée au fait que les actionnaires ne connaissent pas exactement la rentabilité de la firme, Bhattacharya (1979) relève deux coûts associés à l'activité de signalisation : le coût de l'imposition des dividendes supérieur aux gains en capital et le coût relevant de l'endettement contracté par le gestionnaire pour honorer ses engagements à l'égard des actionnaires, au cas où les dividendes promis seraient supérieurs aux bénéfices de la firme. En revanche, Bhattacharya (1980) abandonne l'aspect fiscalité comme élément dissuasif coûteux. Il envisage l'hypothèse où l'activité de signalisation possède un coût de pénalité différent du coût lié à l'endettement contracté pour compenser l'écart entre le dividende attendu et l'insuffisance des bénéfices. Cette pénalité est déterminée par un mécanisme d'ajustement par le marché. Se référant au marché du travail ${ }^{3}$, Bhattacharya propose que la valeur de liquidation de l'entreprise soit ajustée, dans une période donnée, par le marché en fonction de la différence qui apparaîtra entre le dividende annoncé et le premier résultat constaté. Sur cette base, il montre que la valeur de liquidation de l'entreprise est une fonction croissante du dividende versé.

3. Bhattacharya montre que, sur le marché du travail, lorsqu'un salarié déclare ex ante un niveau de productivité, il obtient un salaire fixe en fonction de ce niveau déclaré, si cette productivité est égale ou supérieure à ce qu'il avait annoncé. Dans le cas contraire, le salaire sera diminué d'une pénalité en fonction de l'écart constaté.

Revue internationale P.M.E., vol. 15, nº 1, 2002 


\subsubsection{Les modèles à mécanismes incitatifs}

Contrairement aux modèles à signaux financiers où c'est l'emprunteur qui signale la qualité de son projet au créancier à travers un certain nombre de variables de comportement, les modèles à mécanismes incitatifs sont ceux où c'est plutôt le créancier qui introduit dans les contrats de prêt des éléments qui incitent l'emprunteur à révéler sa qualité. Au rang de ces éléments figurent les garanties, les taux d'intérêt et la relation de clientèle.

En ce qui concerne les garanties, plusieurs auteurs en ont fait l'objet de leurs études. Les principales contributions sont celles de Wette (1983), Besanko et Thakor (1985), Chan et Kanatas (1985), Stiglitz et Weiss (1985), Bester (1987) et Chan et Thakor (1987). Dans la quasi-totalité de ces modèles, les auteurs fondent leur argumentation sur les deux pouvoirs qu'aurait la garantie, à savoir un pouvoir dissuasif, qui limite l'aléa moral, et un pouvoir autosélectif, qui réduit la sélection contraire. La garantie et le taux d'intérêt sont considérés ici comme un coût dans la mesure où, à un risque plus élevé, correspondrait une garantie et / ou un taux d'intérêt plus important. Concernant le pouvoir dissuasif, l'exigence des garanties élevées par le prêteur obligerait l'emprunteur à ne pas détourner le crédit obtenu vers des utilisations autres que le projet initial. Pour ce qui est du pouvoir autosélectif, les garanties et les taux d'intérêt élevés exigés par le prêteur amènent les seuls emprunteurs à risque élevé à solliciter des crédits, car les emprunteurs moins risqués se retirent purement et simplement du marché.

La théorie financière propose aussi l'instauration des relations de clientèle comme un moyen pour contrôler le risque de l'emprunteur. Il s'agit ici d'utiliser les relations de long terme, qui peuvent s'établir dans le temps entre le créancier et l'emprunteur pour réduire le risque moral et la sélection contraire. Ces relations procèdent de l'observation du comportement de l'emprunteur au cours des périodes et se fondent sur les engagements, les promesses et la confiance des parties contractantes. Très souvent, il s'agit de lier ou de rendre le comportement du débiteur temporellement ou intertemporellement cohérent.

Ces relations peuvent s'établir à partir de deux types de contrats : les contrats implicites et les contrats explicites.

\section{a) Les contrats implicites}

Les premiers travaux consacrés aux contrats implicites remontent au début des années 1970 avec notamment les contributions de Azariadis (1975), Bull (1983), Grossman et Hart (1981) et Azariadis et Stiglitz (1983). Dans ces études, la théorie des contrats implicites est applicable en particulier aux relations entre l'employeur

Revue internationale P.M.E., vol. 15, nº 1, 2002 
et les employés sur le marché du travail. Par la suite, les travaux s'intéressent également au marché du crédit, avec Stiglitz et Weiss (1983), Diamond (1989, 1991), Sharpe (1990), Cole (1998), etc.

La théorie financière précise que, lorsqu'il n'existe plus de moyen légal de contraindre l'emprunteur à révéler sa qualité, lorsque le recours à un contrat contingent est trop coûteux, les relations implicites qui existent naturellement entre le banquier et son client peuvent permettre de contourner le problème de la sélection contraire ou de l'aléa moral.

Les clauses du contrat implicite des prêts ne font pas ressortir de manière claire et précise les dispositions - sanctions à l'encontre de l'emprunteur, attitude ou résolutions du banquier à l'égard de son client - à envisager lorsque ces clauses ne sont pas respectées par l'une des parties, en particulier le débiteur. En un mot, les mécanismes d'incitation de l'emprunteur n'apparaissent pas formellement dans les dispositions du contrat de crédit. Les rapports qui s'établissent entre le banquier et son client, du fait de l'opération de crédit demeurent sous-entendus et se basent en général sur deux éléments : la réputation et la bonne foi des parties. Cole (1998), dans son étude empirique, montre que la longévité d'une relation entre le prêteur et l'emprunteur renforcée par différents services financiers entretenus entre ces derniers est positivement corrélée à la décision d'octroi de crédit.

Contrairement à Sharpe (1990), Diamond (1991), Petersen et Rajan (1995) pour qui la relation de long terme devrait se solder par la réduction du coût de crédit accordé, Cole (1998), quant à lui, insiste sur la disponibilité du crédit qu'entraînerait l'existence d'une telle relation.

Les contrats implicites mettent ainsi en jeu la réputation des protagonistes en présence et s'appuient, pour ainsi dire, sur la confiance mutuelle. Cette réputation est importante, car il est possible de recourir à l'observation directe de la qualité de l'emprunteur avant de procéder au choix. Parlant de la réputation du banquier, Sharpe (1990) souligne que, si dans une relation de crédit, les banques exploitent leurs premiers clients, il faudrait vraisemblablement s'attendre à ce qu'elles perdent des parts de marché potentiel sur la nouvelle clientèle, au cas où elle en viendrait à s'informer sur de telles pratiques. Mais dans la plupart des modèles (Diamond, 1989, 1991) par exemple, la réputation du banquier est considérée comme une donnée constante au contraire de celle de l'emprunteur qui, elle, est une variable déterminante.

Au total, les contrats implicites par les effets de réputation et de confiance qu'ils mettent en jeu peuvent être un moyen de limitation du risque de crédit. Toutefois, un certain nombre de réserves doivent être faites sur les principales hypothèses explicatives retenues. D'une manière générale, pour que les effets de réputation jouent de manière significative, il faut au moins deux conditions :

Revue internationale P.M.E., vol. 15, $\mathrm{n}^{\circ}$ 1, 2002 
- que l'agent investisse dans sa réputation et que celle-ci affecte et améliore les revenus et les profits futurs de l'emprunteur. Dans l'hypothèse contraire, où la réputation ne constitue pas une préoccupation pour l'emprunteur - cas des emprunteurs factices opportunistes -, il faudrait s'attendre à ce que l'adoption d'une telle mesure ne produise que des effets limités ;

- que le comportement passé soit un indicateur du comportement d'aujourd'hui et celui d'aujourd'hui, un indicateur du comportement futur. En effet, la réputation est établie à partir de l'observation du comportement passé que l'on projette sur le présent puis sur le futur. Ainsi, pour que la réputation du débiteur se conserve dans le présent et dans l'avenir, il faut supposer un comportement corrélé ou un comportement cohérent et rationnel dans le temps.

Dès lors, la question intéressante est la suivante : comment inciter un emprunteur à préférer une relation de long terme à des contrats à court terme renouvelés qui laissent la porte ouverte à un comportement opportuniste et obligent à une vérification coûteuse du résultat à chaque période?

Ces observations restent dans leur ensemble valables pour les modèles de contrats explicites.

\section{b) Les contrats explicites}

À la différence des contrats implicites, les dispositions des contrats explicites font apparaître clairement, en plus des éléments entrant dans un contrat normal de crédit, des sanctions - résiliation du contrat, exclusion du marché de crédit et rationnement du crédit - auxquelles s'exposent les emprunteurs en cas de non-respect des termes du contrat. Ces contrats explicites sont assortis d'un acte juridique légal donnant droit, entre autres, aux poursuites judiciaires à l'encontre de l'emprunteur défaillant.

Dans la perspective des relations de long terme, Stiglitz et Weiss (1983) envisagent la possibilité pour une banque d'offrir des contrats explicites à deux périodes. Examinant les conditions sous lesquelles il est souhaitable d'utiliser un contrat contingent et celles sous lesquelles un contrat contingent entraîne la dénonciation de la relation, les auteurs aboutissent à la conclusion selon laquelle, dans l'hypothèse de l'incapacité de l'emprunteur à effectuer son remboursement à la fin de la première période, il peut être rationné par le banquier à la deuxième période ; celui-ci peut, par exemple, lui infliger un taux d'intérêt élevé. La solution préconisée par Allen (1983) est bien plus sévère, car il propose l'exclusion de l'emprunteur du marché des crédits en cas de défaillance.

Revue internationale P.M.E., vol. 15, nº 1, 2002 
Mais, qu'il s'agisse des contrats explicites ou des contrats contingents, le principe à la base des analyses et les conditions requises pour l'obtention d'un nouveau crédit sont les mêmes.

En effet, ces modèles se fondent sur l'idée voulant que la menace de suspension des relations de crédit à long terme et l'application des sanctions constituent des mesures incitatives pour l'emprunteur. En outre, le principe de l'antériorité des dettes conditionne les autres crédits. De ce fait, les dettes antérieures ont préséance ou priorité sur les nouvelles dettes. Autrement dit, tout emprunteur ne peut obtenir un autre crédit que s'il s'est acquitté de tous les engagements pris à la première période. Il faut dire que le principe de l'antériorité de la dette est de nature à rendre la menace de suspension ou de rationnement efficace. Toutefois, pour que ces conditions et cette menace soient effectives, il faut au moins deux préalables : l'absence d'autres sources de financement et la menace de suspension des relations de crédit pouvant affecter les profits futurs.

En ce qui concerne l'absence d'autres sources de financement, les emprunteurs potentiels ne doivent pas avoir la possibilité de substituer au crédit bancaire une autre source de financement. Si tel est le cas, ils pourraient rester indifférents à la menace de suspension de crédit ou d'exclusion du marché de crédit. Or, dans le contexte des économies contemporaines et notamment occidentales, où l'accès au marché des capitaux est relativement aisé et banalisé, l'instrument «menace » perd toute sa force en tant que mécanisme incitatif pour l'emprunteur avide de gains, car il peut recourir à ces marchés de capitaux pour se procurer les ressources dont il a besoin. Il est également important de relever que même dans la plupart des pays de l'Afrique subsaharienne où ces marchés sont en général quasi inexistants la présence des circuits financiers informels (tontines et autres associations d'épargne et de crédit) qui accordent les crédits contribuerait à affaiblir cet instrument.

Pour ce qui est de la menace de suspension des relations de crédit pouvant affecter les profits futurs, il convient de signaler que lorsque l'emprunteur n'a pas de profit futur à préserver, il faudrait s'attendre à ce qu'une telle pénalisation n'ait que des effets incitatifs limités sur son comportement et qu'elle ne décourage pas en tant que tel ce dernier dans sa prise de risque.

Au total, les relations de long terme qui peuvent s'établir, soit par des contrats implicites ou des contrats explicites, mettent en jeu la réputation de l'emprunteur pouvant être appréhendée par la régularité dans le remboursement des prêts antérieurs. Ces contrats se fondent sur l'idée selon laquelle la menace de suspension de cette relation et l'application des sanctions constituent un instrument supplémentaire à côté des garanties et, partant, un moyen de réduire la sélection adverse et l'aléa moral, autrement dit, de limiter le risque de crédit. La suspension de la relation 
qui est la conséquence d'une perte de confiance, de réputation et de profit oblige l'emprunteur à rembourser convenablement ses dettes et à adopter un comportement raisonnable dans la gestion du crédit obtenu.

Outre les signaux financiers et les mécanismes incitatifs analysés dans les modèles théoriques, la spécificité du contexte de notre étude nous a amenés à introduire d'autres signaux pouvant contribuer à réduire l'asymétrie de l'information sur le marché de crédit. Il s'agit, notamment, de la rentabilité du projet, de la capacité de remboursement du promoteur et de l'engagement de l'emprunteur envers les tiers (tontines et associations diverses, fournisseurs, centrale des risques, État et organismes sociaux). C'est au test empirique de tous ces éléments qu'est consacrée la deuxième partie de notre travail.

\section{Les déterminants de la politique d'offre de crédit bancaire aux PME camerounaises : une analyse empirique}

Dans cette partie du travail, il s'agit d'identifier, à travers une étude sur le terrain, les variables financières sur lesquelles se basent les banquiers dans leur décision d'octroi de crédit. Faute de pouvoir obtenir directement auprès des banques des données quantitatives sur les crédits octroyés avec des conditions y afférentes, en raison du secret bancaire, nous avons opté pour une enquête sous-tendue par un questionnaire semi-directif. Cette enquête a été effectuée auprès de quelques responsables des principales banques du Cameroun afin de recueillir leur appréciation sur les variables déterminantes de leur décision. La présentation de la méthodologie du travail (2.1.) sera suivie de l'analyse des résultats (2.2).

\subsection{Approche méthodologique}

L'approche méthodologique consistera ici à décrire les variables retenues, l'échantillon sur lequel a porté l'enquête ainsi que la technique statistique utilisée.

\subsubsection{Les variables de l'analyse}

Pour les besoins de l'analyse, nous avons essayé de répertorier les variables financières internes et externes à l'entreprise emprunteuse. Les entretiens auprès des professionnels de la banque nous ont permis de retenir les variables suivantes:

APP : apport personnel du promoteur ${ }^{4}$;

4. Selon les banquiers, cet apport personnel est de $40 \%$ au moins du montant du crédit sollicité. Il représente tout ou partie des capitaux propres du promoteur ôtés des actifs fictifs. 
AFI : autonomie financière du promoteur (niveau des capitaux propres / total des dettes);

RPE : rentabilité du projet (résultat net/investissement en actif);

RDA : régularité des dividendes versés aux actionnaires;

VGA : valeur des garanties apportées ${ }^{5}$;

CRE : capacité de remboursement du promoteur (capacité d'autofinancement / dettes financières);

NEF : niveau d'engagement du promoteur à l'égard des fournisseurs ;

NEC: niveau d'engagement du promoteur à la centrale des risques de la Banque centrale;

VDS : versement régulier des dettes sociales;

PRI : paiement régulier des impôts et taxes;

NTA : niveau d'engagement auprès des tontines et associations ;

RPA : régularité dans le remboursement des prêts antérieurs.

Des 12 variables retenues, la variable RPA permet d'appréhender la relation de long terme ou de clientèle.

Il s'agira d'apprécier le poids de chacune de ces variables dans la décision d'octroi des crédits.

\subsubsection{Constitution et description de l'échantillon}

Notre étude a porté sur un échantillon de 8 banques sur 12 établissements de crédit que compte actuellement le Cameroun. Nous avons fait prévaloir dans le choix le critère d'ancienneté; c'est ainsi que les banques nouvellement créées n'ont pas été retenues. Des huit banques de l'échantillon, trois sont des filiales de groupes étrangers parmi lesquelles une banque d'affaires et les cinq autres sont des groupes à capitaux nationaux prédominants. La faible densité du réseau bancaire camerounais, donc de l'échantillon, nous a conduit à solliciter des entretiens auprès des cadres des services des engagements afin de réduire la subjectivité des réponses obtenues. Sur une trentaine de questionnaires adressés à ces derniers, seuls 21 nous ont répondu, ce qui donne un taux de réponse de $70 \%$. Notre analyse a ainsi porté sur ces 21 cas.

5. Les garanties sont représentées ici par la valeur des hypothèques, des nantissements, des cautions et avals de toutes sortes. 


\subsubsection{Méthode statistique}

Devant un grand nombre de données multivariées supposées influencer la décision du banquier, nous avons retenu l'analyse en composantes principales comme méthode statistique dans le but de résumer l'information apportée par un grand nombre de variables, par un nombre plus restreint de variables nouvelles. La plupart des variables étant liées, cette méthode consiste à concevoir à partir d'un tableau «individus-variables» de données quantitatives un autre tableau «individusnouvelles variables », en réduisant le nombre de variables nécessaires pour décrire les individus, tout en perdant le moins possible d'information.

Le questionnaire adressé aux banquiers les invitait, en fonction des variables retenues dans chaque question, à attribuer une note allant de 1 à 5 eu égard à l'importance de la variable dans la décision d'octroi de crédit.

À partir de l'analyse en composantes principales, nous avons effectué deux tests: un premier sur les variables financières hormis les relations de long terme ou de clientèle; un deuxième sur les variables financières avec prise en compte des relations de long terme.

Le choix des axes ou composantes principales est fait en fonction d'un seuil correspondant au pourcentage minimal d'inertie que nous souhaitions restituer. Pour cela, on a retenu le nombre $q$ d'axes nécessaires pour atteindre ce seuil que nous avons fixé au moins à $80 \%$. Ce seuil est égal au rapport entre la somme des valeurs propres et la trace de la matrice des variances-covariances de l'espace vectoriel considéré W. Il est donné par la formule suivante :

$$
\mathrm{I}_{\mathrm{W}}=\left[\left(\lambda_{1}+\lambda_{2}+\ldots+\lambda_{\mathrm{q}}\right) / \text { trace } \mathrm{V}\right] \geq 80 \%
$$

où :

$\lambda_{1}+\lambda_{2}+\ldots+\lambda_{\mathrm{q}}$ représentent les valeurs propres;

$\mathrm{V}$ représente la matrice de variance-covariance de la matrice individusvariables;

$\mathrm{I}_{\mathrm{W}}$ représente l'inertie expliquée.

\subsection{Analyse des résultats}

Cette partie du travail est consacrée à la présentation des résultats de l'étude (2.2.1.) et à leur interprétation (3.2.2.). 


\subsubsection{Présentation des résultats de l'étude}

Le traitement de nos données a été réalisé avec le logiciel $S p a d^{6}$. Les résultats obtenus sont les suivants. Les valeurs propres issues des deux tests sont données respectivement par le tableau 1 (variables financières, hormis la relation de long terme) et le tableau 2 (variables financières avec relation de long terme) ci-après.

TABLEAU 1

Histogramme des valeurs propres (sans relation de long terme)

\begin{tabular}{lcccc}
\hline Variables & $\begin{array}{c}\text { Numéros } \\
\text { des axes }\end{array}$ & $\begin{array}{c}\text { Valeurs } \\
\text { propres }\end{array}$ & $\begin{array}{c}\text { Pourcentage } \\
\text { d'inertie expliquée }\end{array}$ & $\begin{array}{c}\text { Pourcentage } \\
\text { cumulé }\end{array}$ \\
\hline APP & 1 & 5,5306 & 50,28 & 50,28 \\
AFI & 2 & 2,3337 & 21,22 & 71,49 \\
RPE & 3 & 1,5573 & 14,16 & 85,65 \\
RDA & 4 & 1,1707 & 10,64 & 96,29 \\
VGA & 5 & 0,2542 & 2,31 & 98,60 \\
CRE & 6 & 0,1148 & 1,04 & 99,65 \\
NEF & 7 & 0,0387 & 0,35 & 100,00 \\
NEC & 8 & 0,0000 & 0,00 & 100,00 \\
VDS & 9 & 0,0000 & 0,00 & 100,00 \\
PRI & 10 & 0,0000 & 0,00 & 100,00 \\
NTA & 11 & 0,0000 & 0,00 & 100,00 \\
\hline
\end{tabular}

TABLEAU 2

Histogramme des valeurs propres (avec relation de long terme)

\begin{tabular}{lcccc}
\hline Variables & $\begin{array}{c}\text { Numéros } \\
\text { des axes }\end{array}$ & $\begin{array}{c}\text { Valeurs } \\
\text { propres }\end{array}$ & $\begin{array}{c}\text { Pourcentage } \\
\text { d'inertie expliquée }\end{array}$ & $\begin{array}{c}\text { Pourcentage } \\
\text { cumulé }\end{array}$ \\
\hline APP & 1 & 6,0812 & 50,68 & 50,68 \\
AFI & 2 & 2,3859 & 19,88 & 70,56 \\
RPE & 3 & 1,8983 & 15,82 & 86,38 \\
RDA & 4 & 1,1948 & 9,96 & 96,33 \\
VGA & 5 & 0,2755 & 2,30 & 98,63 \\
CRE & 6 & 0,1154 & 0,96 & 99,59 \\
NEF & 7 & 0,0490 & 0,41 & 100,00 \\
NEC & 8 & 0,0000 & 0,00 & 100,00 \\
VDS & 9 & 0,0000 & 0,00 & 100,00 \\
PRI & 10 & 0,0000 & 0,00 & 100,00 \\
NTA & 11 & 0,0000 & 0,00 & 100,00 \\
RPA & 12 & 0,0000 & 0,00 & 100,00 \\
\hline
\end{tabular}

6. Spad version 1998 est un logiciel économétrique de traitement des données statistiques. 
On observe que les trois premiers axes suffisent pour expliquer ces tableaux de données puisqu'ils totalisent respectivement $85,65 \%$ de l'inertie pour le tableau 1 et $86,38 \%$ pour le tableau 2 , le seuil minimal fixé dans l'analyse étant de $80 \%$.

Les matrices des corrélations sont données par les tableaux 3 et 4 ci-après.

TABlEAU 3

Matrice des corrélations (sans relation de long terme)

\begin{tabular}{lrrrrrrrrrrr}
\hline & APP & AFI & RPE & RDA & VGA & CRE & NEF & NEC & VDS & PRI & NTA \\
\hline APP & 1,00 & & & & & & & & & & \\
AFI & 0,69 & 1,00 & & & & & & & & & \\
RPE & $-0,15$ & $-0,15$ & 1,00 & & & & & & & & \\
RDA & $-0,19$ & 0,14 & $-0,06$ & 1,00 & & & & & & & \\
VGA & 0,67 & 0,04 & 0,30 & $-0,52$ & 1,00 & & & & & & \\
CRE & $-0,05$ & $-0,40$ & 0,86 & $-0,34$ & 0,57 & 1,00 & & & & & \\
NEF & 0,17 & $-0,47$ & 0,49 & $-0,51$ & 0,76 & 0,75 & 1,00 & & & & \\
NEC & 0,27 & $-0,25$ & 0,43 & $-0,65$ & 0,67 & 0,68 & 0,79 & & & & \\
VDS & 0,10 & $-0,09$ & $-0,73$ & 0,44 & $-0,16$ & $-0,64$ & $-0,17$ & $-0,39$ & 1,00 & & \\
PRI & 0,42 & 0,79 & $-0,56$ & 0,53 & $-0,35$ & $-0,79$ & $-0,74$ & $-0,67$ & 0,43 & 1,00 & \\
NTA & $-0,09$ & 0,27 & $-0,12$ & 0,93 & $-0,47$ & $-0,41$ & $-0,54$ & $-0,81$ & 0,44 & 0,65 & 1,00 \\
\hline
\end{tabular}

TABLEAU 4

Matrice des corrélations (avec relation de long terme)

\begin{tabular}{lrrrrrrrrrrrr}
\hline & APP & AFI & RPE & RDA & VGA & CRE & NEF & NEC & VDS & PRI & NTA & RP \\
\hline APP & 1,00 & & & & & & & & & & & \\
AFI & 0,69 & 1,00 & & & & & & & & & & \\
RPE & $-0,15$ & $-0,15$ & 1,00 & & & & & & & & & \\
RDA & $-0,19$ & 0,14 & $-0,06$ & 1,00 & & & & & & & & \\
VGA & 0,67 & 0,04 & 0,30 & $-0,52$ & 1,00 & & & & & & & \\
CRE & $-0,05$ & $-0,40$ & 0,86 & $-0,34$ & 0,57 & 1,00 & & & & & & \\
NEF & 0,17 & $-0,47$ & 0,49 & $-0,51$ & 0,76 & 0,75 & 1,00 & & & & & \\
NEC & 0,27 & $-0,25$ & 0,43 & $-0,65$ & 0,67 & 0,68 & 0,79 & & & & & \\
VDS & 0,10 & $-0,09$ & $-0,73$ & 0,44 & $-0,16$ & $-0,64$ & $-0,17$ & $-0,39$ & 1,00 & & & \\
PRI & 0,42 & 0,79 & $-0,56$ & 0,53 & $-0,35$ & $-0,79$ & $-0,74$ & $-0,67$ & 0,43 & 1,00 & & \\
NTA & $-0,09$ & 0,27 & $-0,12$ & 0,93 & $-0,47$ & $-0,41$ & $-0,54$ & $-0,81$ & 0,44 & 0,65 & 1,00 & \\
RPA & 0,25 & 0,17 & 0,80 & $-0,39$ & 0,47 & 0,77 & 0,44 & 0,65 & $-0,86$ & $-0,41$ & $-0,44$ & 1,00 \\
\hline
\end{tabular}

Les tableaux 5 et 6 contiennent les coefficients de corrélation entre les variables et les cinq premières composantes principales. Ils vont servir à représenter les variables dans les cercles de corrélation.

À partir des tableaux 5 et 6 , on peut obtenir les figures et les cercles de corrélations associés dans le plan des axes 1 et 2 et des axes 2 et 3 conformément au pourcentage d'inertie expliquée par les trois axes, à savoir 85,65\% pour le tableau 1 et $86,38 \%$ pour le tableau 2 . Ces figures et cercles de corrélations associés sont présentés en annexe. Il s'agit des figures 1 et 2 pour ce qui est du test hormis la relation de long terme et des figures 3 et 4 du test avec relation de long terme.

Revue internationale P.M.E., vol. 15, nº 1, 2002 
TABleau 5

Corrélations variable-facteur (sans relation de long terme)

\begin{tabular}{lrrrrr}
\hline \multirow{2}{*}{$\begin{array}{l}\text { Variables } \\
\text { actives }\end{array}$} & Axe 1 & Axe 2 & Axe 3 & Axe 4 & Axe 5 \\
\cline { 2 - 5 } & 0,04 & 0,97 & 0,12 & $-0,18$ & 0,02 \\
\hline APP & $-0,43$ & 0,72 & 0,46 & 0,26 & 0,04 \\
AFI & 0,63 & $-0,27$ & 0,71 & $-0,09$ & 0,04 \\
RPE & $-0,71$ & $-0,31$ & 0,37 & $-0,46$ & 0,21 \\
RDA & 0,68 & 0,59 & 0,03 & $-0,35$ & $-0,20$ \\
VGA & 0,87 & $-0,18$ & 0,38 & $-0,19$ & $-0,04$ \\
CRE & 0,86 & 0,08 & $-0,12$ & $-0,43$ & $-0,03$ \\
NEF & 0,88 & 0,24 & $-0,11$ & $-0,02$ & 0,38 \\
NEC & $-0,57$ & 0,09 & $-0,58$ & $-0,57$ & 0,06 \\
VDS & $-0,87$ & 0,45 & 0,16 & 0,03 & 0,04 \\
PRI & $-0,78$ & $-0,19$ & 0,40 & $-0,43$ & $-0,10$ \\
NTA & & & &
\end{tabular}

TABleau 6

Corrélations variable-facteur (avec relation de long terme)

\begin{tabular}{lrrrrr}
\hline \multirow{2}{*}{$\begin{array}{l}\text { Variables } \\
\text { actives }\end{array}$} & Axe 1 & Axe 2 & Axe 3 & Axe 4 & Axe 5 \\
\cline { 2 - 6 } & 0,09 & $-0,96$ & $-0,13$ & 0,21 & $-0,02$ \\
\hline APP & $-0,34$ & $-0,84$ & 0,37 & $-0,17$ & 0,00 \\
AFI & 0,69 & 0,16 & 0,65 & 0,22 & 0,02 \\
RPE & $-0,69$ & 0,20 & 0,43 & 0,50 & $-0,22$ \\
RDA & 0,68 & $-0,52$ & $-0,25$ & 0,38 & 0,21 \\
VGA & 0,89 & 0,16 & 0,28 & 0,26 & 0,06 \\
CRE & 0,83 & 0,02 & $-0,29$ & 0,42 & 0,03 \\
NEF & 0,88 & $-0,16$ & $-0,23$ & 0,01 & $-0,37$ \\
NEC & $-0,65$ & 0,02 & $-0,60$ & 0,45 & $-0,12$ \\
VDS & $-0,83$ & $-0,53$ & 0,15 & $-0,01$ & $-0,04$ \\
PRI & $-0,75$ & 0,08 & 0,43 & 0,48 & 0,09 \\
NTA & 0,78 & $-0,28$ & 0,53 & $-0,10$ & $-0,12$ \\
RPA & & & & & \\
\hline
\end{tabular}

\subsubsection{Interprétation des résultats}

L'interprétation des résultats sera fonction des composantes principales représentées par les trois axes retenus.

En ce qui concerne le test portant sur les variables financières hormis la relation de long terme, il apparaît que l'axe 1 ou première composante principale peut être interprété comme niveau d'engagement de l'emprunteur envers les 
tiers. Cela est confirmé par le tableau 5 des corrélations où les variables NEF et NEC représentant respectivement les niveaux d'engagements envers les fournisseurs et à la centrale des risques, bien représentées sur le cercle de la figure 1, sont celles les plus corrélées avec l'axe 1.

Si on appelle $r$ ce coefficient de corrélation, on a bien :

$$
\begin{array}{lll}
\mathrm{r}_{\mathrm{NEF} \text { axe } 1}=0,86 & \mathrm{r}_{\mathrm{NEF} \text { axe } 2}=0,08 & \mathrm{r}_{\mathrm{NEF} \text { axe3 }}=-0,12 \\
\mathrm{r}_{\mathrm{NEC} \text { axe } 1}=0,88 & \mathrm{r}_{\mathrm{NEC} \mathrm{axe} 2}=0,24 & \mathrm{r}_{\mathrm{NEC} \mathrm{axe} 3}=-0,11
\end{array}
$$

D'après ces résultats, il est clair que, dans leur politique d'octroi des crédits, les banquiers tiennent compte du niveau d'engagement de l'emprunteur déterminé par les variables NEF et NEC qui ont une incidence certaine sur sa capacité de remboursement CRE, fortement corrélée avec l'axe 1. Un niveau élevé de ces deux variables réduit la capacité de remboursement de l'emprunteur. Autrement dit, plus le niveau d'engagement de l'emprunteur envers les tiers est important, plus la banque serait réticente à lui accorder son concours. Il est apparu à ce niveau que la plupart des banques à prédominance de capitaux nationaux sont beaucoup plus portées vers ces critères. Toutefois, en l'absence de réglementation sur le niveau d'engagement de l'emprunteur envers les tiers, il revient à chaque banque d'apprécier le seuil critique au-delà duquel toute demande de crédit est refusée.

En revanche, les variables telles que PRI, NTA et VDS, représentant respectivement le paiement régulier des impôts et taxes, le niveau d'engagement auprès des associations et le versement régulier des dettes sociales, sont négativement corrélées avec cette composante principale; ce qui signifie qu'elles sont moins considérées à ce niveau par les banquiers.

Par ailleurs, les banquiers sont dans l'impossibilité de rassembler les informations fiables concernant l'engagement des PME envers les associations ainsi que les autres informations concernant le paiement des impôts et dettes sociales.

La deuxième composante principale (axe 2) peut être interprétée comme l'importance du patrimoine de l'emprunteur. Cela se traduit par la forte corrélation positive de la variable APP (apport personnel du promoteur) avec l'axe 2 de la figure 1 , d'après le coefficient de corrélation qui est:

$$
\mathrm{r}_{\mathrm{APP} \text { axe2 }}=0,97 \quad \mathrm{r}_{\mathrm{APP} \text { axe } 1}=0,04 \quad \mathrm{r}_{\mathrm{APP} \text { axe } 3}=0,12
$$

Ce résultat révèle que l'apport personnel est un élément déterminant de la décision du banquier. Cette position est confortée par l'autonomie financière (AFI) et la valeur de la garantie apportée (VGA) par l'emprunteur dont les coefficients de corrélation, positifs et significatifs, sont respectivement de 0,72 et 0,59 . Ce critère revêt une importance capitale pour toutes les banques commerciales de l'échantillon en dehors de l'unique banque d'affaires où il paraît secondaire. 
La variable rentabilité du projet (RPE) est bien représentée et elle est fortement corrélée positivement avec la troisième composante principale (axe 3 de la figure 2). Cet axe peut ainsi être assimilé à la rentabilité du projet de l'emprunteur. Le coefficient de corrélation de la variable RPE est donc:

$$
\mathrm{r}_{\mathrm{RPE} \text { axe3 }}=0,71 \quad \mathrm{r}_{\mathrm{RPE} \text { axe2 }}=-0,27 \quad \mathrm{r}_{\mathrm{RPE} \text { axe1 }}=0,63
$$

Cela montre que l'attention du banquier peut être portée sur un projet rentable dont le promoteur présente un faible niveau de garanties. L'étude révèle que ce critère semble être une caractéristique de la banque d'affaires puisque c'est la seule banque de l'échantillon pour laquelle ce critère occupe une place de choix dans la décision d'octroi de crédit, les autres banques privilégiant la garantie comme déjà relevé. Cela confirme l'optique des banques d'affaires dont la logique est de financer des projets à long terme rentables. Cet état de choses expliquerait également la méfiance des banques commerciales à l'égard du risque, eu égard aux nombreuses faillites bancaires de la décennie écoulée, et leur préférence pour la garantie aux dépens de la rentabilité à terme des projets.

Pour ce qui est du test avec prise en compte de la relation de long terme, on note que la composante principale (axe 1 de la figure 3 ) garde sa dénomination de niveau d'engagement de l'emprunteur envers les tiers d'après le tableau 6 des corrélations. Cela est conforté par la variable relation de clientèle représentée par RPA (régularité dans le remboursement des prêts antérieurs). Cette variable, dont le coefficient de corrélation est 0,78 , est fortement liée positivement à l'axe 1 .

En revanche, l'axe 2 de la figure 3 représente la moindre importance du patrimoine de l'emprunteur. Cela est plus confirmé par la variable apport personnel du promoteur (APP), dont le coefficient de corrélation négatif est plus important par rapport à l'axe 2 :

$$
\mathrm{r}_{\mathrm{APP} \text { axe } 2}=-0,96 \quad \mathrm{r}_{\mathrm{APP} \text { axe } 1}=0,09 \quad \mathrm{r}_{\mathrm{APP} \text { axe } 3}=-0,13
$$

Toutefois, lorsqu' on tient compte du critère « relation de long terme » (RPA), les variables APP (apport personnel), AFI (autonomie financière) et VGA (valeur des garanties), bien que fortement corrélées avec l'axe 2 de la figure 3 , sont de signe négatif, comme le montrent leurs coefficients de corrélation respectifs ( $c f$. tableau 6). Ces résultats révèlent que, bien que ces variables soient bien représentées, la prise en compte de la réputation de l'emprunteur appréhendée par la régularité dans le remboursement des prêts antérieurs (RPA) relègue au second plan l'importance du patrimoine mis en gage par le promoteur à l'appui de sa demande de crédit. Cela confirme l'idée classique selon laquelle un patrimoine important en gage d'un emprunt ne signifie pas automatiquement un remboursement assuré, vu les difficultés qu'éprouvent très souvent les banquiers à réaliser la garantie en cas de défaillance de l'emprunteur. 
La variable RPE (rentabilité du projet) significativement et positivement corrélée avec l'axe 3 de la figure 4 confirme son nom de rentabilité du projet de l'emprunteur ( $c f$. tableau 6). Cette rentabilité va de pair avec la régularité dans le remboursement des prêts antérieurs (RPA), elle aussi, bien représentée sur l'axe 3. Ce qui laisse penser que le choix de projet rentable incite l'emprunteur de bonne moralité à honorer régulièrement ses engagements. Ce résultat valide les conclusions des modèles théoriques à mécanismes incitatifs (Diamond, 1989, 1991; Sharpe, 1990) selon lesquels les emprunteurs qui investissent dans leur réputation signalent leur qualité dans le choix des projets moins risqués. Ce qui limite à la fois la sélection adverse et l'aléa moral.

Qu'il s'agisse du premier ou du second test, on note que la variable RDA (régularité des dividendes versés aux actionnaires) est fortement corrélée négativement avec la composante principale niveau d'engagement de l'emprunteur envers les tiers. Son coefficient de corrélation pour les premier et second tests est respectivement de $-0,71$ et de $-0,69$. Ce résultat traduit le fait que les banques camerounaises accordent peu d'importance à ce critère dans leur décision. Cela confirme notre hypothèse selon laquelle le versement régulier des dividendes ne saurait être un bon signal pour le banquier, dans le contexte camerounais où les promoteurs des PME sont généralement actionnaires majoritaires de leurs affaires. Dans une telle situation, le pouvoir de contrôle et de décision revient au seul chef d'entreprise. Ainsi, retenir la distribution des dividendes comme indicateur de risque n'aurait qu'une portée significative limitée. Ce qui rejoint ici les conclusions des analyses de Downes et Heinkel (1982) infirmant ainsi celles de Bhattacharya (1979, 1980) et de Kalay (1980).

\section{Conclusion}

De l'analyse précédente, il ressort que l'information financière tant interne qu'externe à l'entreprise revêt une importance capitale dans la décision d'octroi de crédit aux PME par les banques camerounaises.

Sur le plan théorique, il existe deux grands groupes de modèles susceptibles de réduire les imperfections de l'information financière. C'est le cas, d'une part, des modèles à signaux financiers (apport personnel, structure financière, dividendes versés) et, d'autre part, des modèles à mécanismes incitatifs (garanties, relation de

long terme). À cela s'ajoutent les formules de capital-risque qui, ces derniers temps, sont au centre de préoccupations d'un certain nombre d'auteurs en finance d'entreprise.

La spécificité du contexte de notre étude nous a amenés à introduire d'autres signaux pouvant contribuer à réduire l'asymétrie de l'information sur le marché de

Revue internationale P.M.E., vol. 15, nº 1, 2002 
crédit. Il s'agit notamment de la rentabilité du projet, de la capacité de remboursement du promoteur et de l'engagement de l'emprunteur envers les tiers (tontines et associations diverses, fournisseurs, centrale des risques, État et organismes sociaux).

Sur le plan empirique, on note que lorsqu'on ne tient pas compte de la relation de long terme, les informations financières qui entrent dans la décision d'octroi de crédit sont pour l'ensemble des banques de l'échantillon, l'apport personnel du promoteur et le niveau d'engagement des emprunteurs envers les tiers. À cela s'ajoutent la valeur des garanties pour la banque commerciale et la rentabilité du projet pour la banque d'affaires. L'introduction de la relation de clientèle, notamment la régularité dans le remboursement des prêts antérieurs, influence la composante principale «patrimoine de l'emprunteur». Dans le premier test sans relation de clientèle, cette composante revêt une grande importance, tandis que dans le second test avec prise en compte de la relation de clientèle, elle est de moindre importance. Dès lors, il apparaît que l'intégration du critère « relation de long terme » relègue au second plan le patrimoine de l'emprunteur au profit de celle-ci. Cela justifie le fait qu'un patrimoine important n'est pas synonyme d'un remboursement assuré ou une garantie sûre en cas de risque de défaut, étant donné la difficulté qu'éprouve très souvent le banquier à réaliser cette garantie. Autrement dit, au-delà de l'apport personnel de l'emprunteur, variable quantifiable, l'existence d'une relation antérieure qui permet d'établir sa réputation est un critère fondamental dans la décision de prêt.

De manière générale, pour des montants de crédit relativement importants, l'apport personnel de l'emprunteur en termes de ses fonds propres et la relation de long terme sont tous deux pris en compte par le banquier dans sa décision. Mais il convient de noter que la relation de long terme ne joue pas pour les nouveaux clients qui doivent, en plus de l'apport personnel dans le projet et de la garantie apportée, justifier la rentabilité de celui-ci. De même, il y a lieu de signaler que, pour des entreprises nouvellement créées, l'information financière est souvent déficiente ; c'est ainsi que, se référant au capital-risque, pour les investisseurs, les qualités morales de l'entrepreneur et la rentabilité estimée du projet (à partir des éléments prévisionnels) sont mises à contribution.

Dans l'ensemble, les résultats obtenus viennent confirmer, entre autres, un certain nombre de conclusions des modèles théoriques. Toutefois, il convient de mentionner quelques limites. Le nombre limité d'observations et le biais imputable aux enquêtes relié au fait que les répondants ont tendance à mentionner plus de critères qu'ils n'en utilisent dans leurs décisions. La difficulté liée à la détermination d'une variable pouvant permettre d'apprécier de manière exacte les qualités morales de l'emprunteur par le décideur en cas d'information financière déficiente, eu égard aux critères d'investissement dans le cas du capital-risque. Notons cependant que cette étude pourrait constituer une base de réflexion pour les recherches futures. 
AnNeXe I

FIGURE 1

Composantes principales axe 1 / axe 2 (sans relation de long terme)

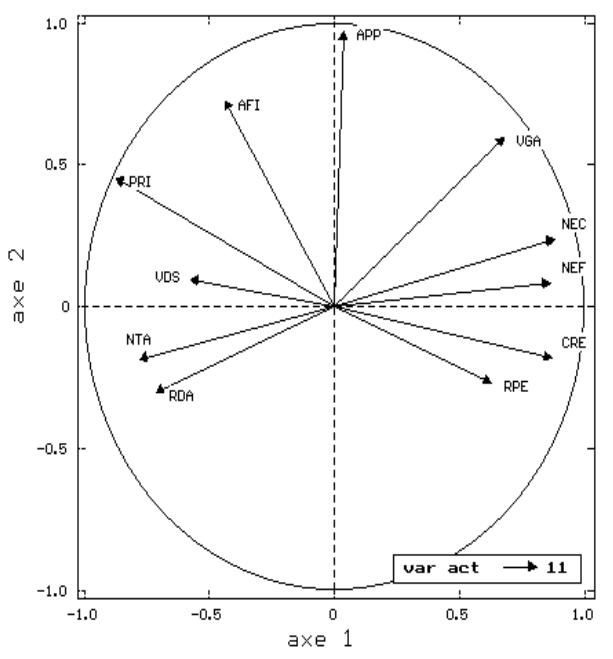

FIGURE 2

Composantes principales axe 2 / axe 3 (sans relation de long terme)

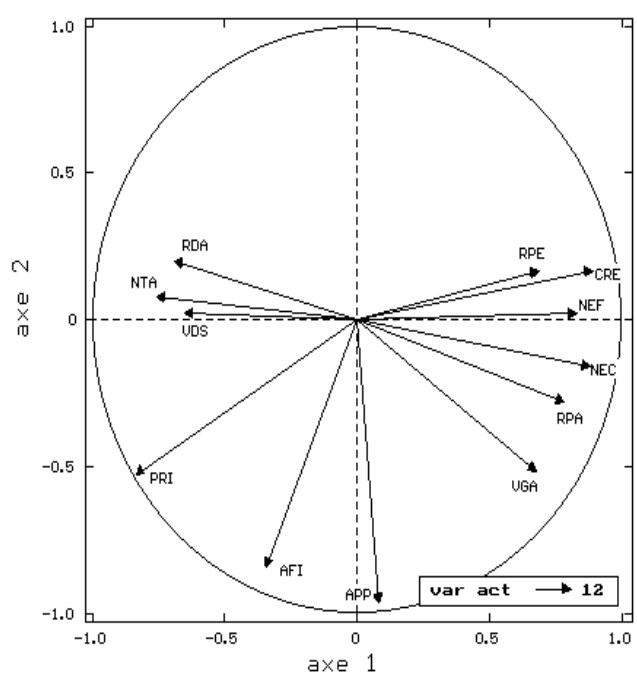

Revue internationale P.M.E., vol. 15, $\mathrm{n}^{\circ}$ 1, 2002 
FigURE 3

Composantes principales axe 1 / axe 2 (avec relation de long terme)

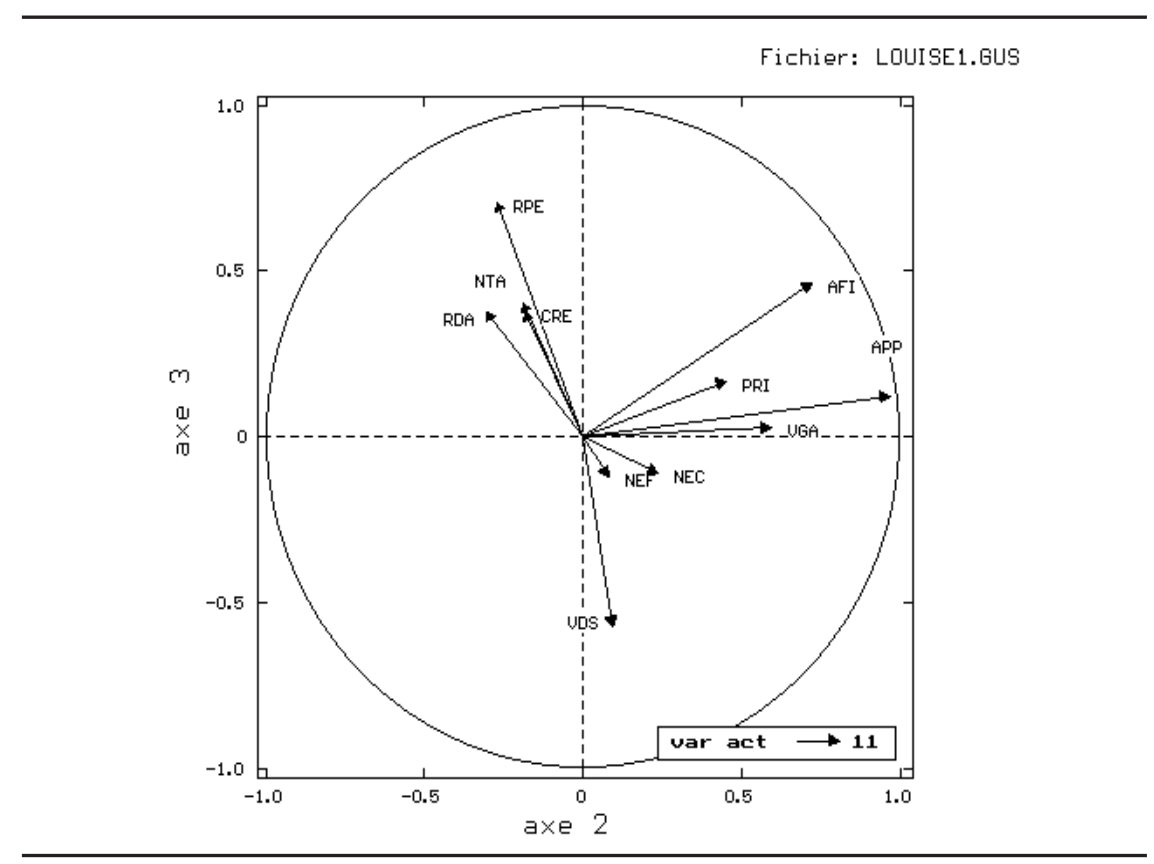

FIGURE 4

Composantes principales axe 2 / axe 3 (avec relation de long terme)

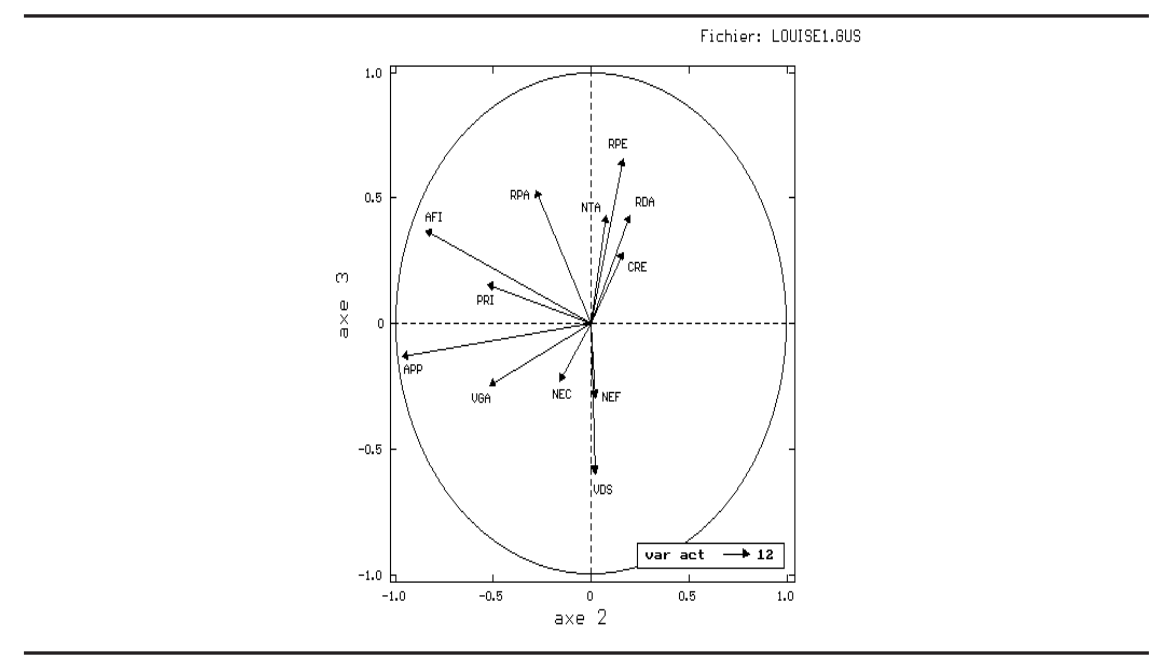

Revue internationale P.M.E., vol. 15, n 1, 2002 


\section{Bibliographie}

AKERLOF, B. (1970), «The market for lemons: quality uncertainty and the market mechanism », Quarterly Journal of Economics, vol. 89, août, p. 488-500.

ALlEN, F. (1983), «Credit rationing and payment incentives », Review of Economic Studies, p. 639-647.

Altmann, E.I. (1980), «Commercial bank lending: process, credit scoring and costs errors lending », Journal of Finance and Quantitative Analysis, vol. 4, novembre, p. 813-832.

AZARIADIS, C. (1975), «Implicit contracts and unemployment equilibria », Journal of Political Economic, décembre, p. 183-202.

AZARIADIS, C. et J.E. STIGLITZ (1983), «Implicits contracts and fixed price equilibria », Quarterly Journal of Economics.

Besanko, D. et A.V. ThAKOR (1985), Competitive Collateral and Rationing Models of Sorting Equilibria in the Credit Market, BRC, Document de travail n ${ }^{\circ}$ 96, Northwestern University.

BESTER, H. (1987), « The role of collateral in credit market with imperfect information », European Economic Review, no 31, p. 887-899.

BHATTACHARYA, S. (1979), «Imperfect information, dividend policy and "the bird in the hand" fallacy », Bell Journal of Economics, vol. 10.

BHATTACHARYA, S. (1980), «Non dissipative signaling structures and dividend policy », Quarterly Journal of Economics, vol. 1, août.

BULL, C. (1983), «Implicit contract in the absence of enforcement and risk aversion», American Economic Review, vol. 83, septembre, p. 658-671.

CHAN, Y. et G. KANATAS (1985), «Asymetric valuations and the role of collateral in loan agreements », Journal of Money, Credit and Banking, vol. 17, février, p. 84-95.

CHAN, Y.S. et A.V. THAKOR (1987), « Collateral and competitive equilibrium with moral hazard and private information », Journal of Finance, vol. XLII, n ${ }^{\circ}$ 2, juin, p. 345-363.

COLE, R.A. (1998), « The importance of relationships to the availability of credit », Journal of Banking and Finance, vol. 22, p. 959-997.

DARrough, M.N et N.M. Stoughton (1986), «Moral hazard and adverse selection: the question of financial structure », Journal of Finance, vol. XLI, n 2, juin, p. 501-513.

Diamond, D.W. (1989), «Reputation acquisition in debt market», Journal of Political Economy, août, p. 828-862.

DiAMOND, D.W. (1991), « Monitoring and reputation : the choice between bank and directly placed debt », Journal of Political Economy, vol. 99, n 4, p. 689-721.

DownES, D.H. et R. HEINKEL (1982), «Signaling and the valuation of unseasoned new issues », Journal of Finance, vol. XXXVII, n 1, mars, p. 1-10.

GROSSMAN, S.J. et O.D. HART (1981), « Implicits contracts moral hazard and unemployment », American Economic Review, mai, p. 301-307.

KALAY, A. (1980), «Signaling, information content and the reluctance to cut dividends », Journal of Financial and Quantitative Analysis, vol. XV, no 4, novembre.

Revue internationale P.M.E., vol. 15, nº 1, 2002 
LELAND, H et D. PYLE (1977), « Informational asymetric, financial structure and financial intermediation », Journal of Finance, vol. XXXII, n 2, mai, p. 371-387.

LE NoIR, A. (1989), «La restructuration nécessaire des systèmes bancaires africains », Revue Techniques financières et développement, vol. 7, $\mathrm{n}^{\text {os }}$ 28-29, septembre-décembre, p. 73-74.

LE NOIR, A. (1995), «Les systèmes financiers actuels sont-ils adaptés aux besoins de l'Afrique ?», Revue Techniques financières et développement, vol. 7, $\mathrm{n}^{\text {os }} 38-39$, marsjuin, p. 72-74.

MALÉCOT, J.F.(1984), «La mesure empirique des coûts de faillite : une note », Finance, vol. 5, no 2, p. 209-219.

Modigliani, F. et M. Miller (1958), «The cost of capital, corporation finance and the theory of investment », American Economic Review, juin.

Petersen, M.A. et R.G. RaJAn (1995), «The effect of credit market competition on lending relationships », Quarterly Review of Economics, vol. 110, p. 407-442.

RAPPORT DU GROUPE DE TRAVAIL ESF (1990), «La crise bancaire africaine: les causes, les manifestations, les remèdes », Revue Techniques financières et développement, vol. $7, n^{\text {os }} 28-29$, p. 74-88.

Ross, S.A. (1977), «The definition of financial structure: the incentive signaling approach », Bell Journal of Economics, printemps, p. 23-40.

SHARPE, S.A. (1990), «Asymetric information, bank lending and implicit contracts : a stylized model of customer relationships », Journal of Finance, vol. XLV, $n^{\circ} 4$, septembre, p. 1069-1087.

STIGLITZ, J.E. et A. WeISS (1981), «Credit rationing in markets with imperfect information », America Economic Review, juin, p. 393-410.

STIGLITZ, J.E. et A. WEISS (1983), «Incentive effets of termination : application to credit and loans market », American Economic Review, no 73, p. 912-927.

StigLiTZ, J.E. et A. WeISS (1985), « Credit rationing with collateral », Bell Communication Research Inc., Economics Discussion Papers, vol. 12, décembre.

TAMBA, I. et L. TCHAMANBÉ-DJINÉ (1995), « De la crise à la réforme des institutions bancaires africaines : l'expérience du Cameroun », Revue Tiers Monde, tome XXXVI, $\mathrm{n}^{\circ} 144$, octobre-décembre, p. 813-835.

WAMBA, H. (1989), La gestion du risque bancaire en matière d'accord de crédit aux entreprises, Thèse de doctorat, Université de Rennes I, juin, 386 p.

WAmBA, H. (2001), «La gestion bancaire en Afrique centrale à 1'heure des grandes mutations : bilan et perspectives », Revue Gestion 2000, nº 6, novembre-décembre.

WARNER, J.B. (1977), «Bankruptcy : some evidence », Journal of Finance, mai, p. 337-347.

WETTE, H.C. (1983), « Collateral in credit rationing in market with imperfection information: note », American Economic Review, vol. 73, juin, p. 442-445.

Wright, M. et K. RoBbIE (1998), «Venture capital and private equity: a review and syntheses », Journal of Business Finance \& Accounting, vol. 25, $\mathrm{n}^{\mathrm{os}} 5-6$, juin-juillet, p. 521-570. 